\title{
Maladaptive Alterations of Autonomic Nerve System in Cardiovascular Disorders
}

\author{
Yukiteru Nakayama, ${ }^{1}$ MD and Katsuhito Fujiu, ${ }^{1,2}$ MD
}

(Int Heart J 2019; 60: 4-6)

$\mathrm{S}$ udden unexpected nocturnal death syndrome (SUNDS) occurs predominantly in Southeast Asia, and the young healthy victims are struck by unexplained deaths during nocturnal sleep. The predilection time implies that the balance of the autonomic nervous system is associated with arrhythmogenesis. Mongkonsiri, et al. reported that healthy subjects with a family history of SUNDS showed blunted responsiveness to cold pressor testing and steady exercise during the nighttime, ${ }^{1)}$ indicative of decreased sympathetic and increased parasympathetic nerve activity. Regarding the autonomic imbalance in patients with Brugada syndrome, a disease relevant to SUNDS, inconsistent results have been reported. Some papers have revealed that adrenergic dysfunction was observed in diseased groups, while others reported no differences in heart rate variability. ${ }^{2)}$ However, Brugada syndrome and SUNDS are regarded as different diseases in that the latter is more likely a multifactorial disorder caused by both intrinsic pathogenic genes and external environmental factors. ${ }^{3)}$ Various factors implicated with susceptibility to SUNDS, including diet, lifestyle, and work intensity could contribute to the tuning of the autonomic nervous system rather than the direct effects of genetics, as previously reported. ${ }^{4)}$

\section{Article p.55}

Blunting of autonomic reflex by repeated stimuli is not necessarily considered an adaptive response. An acute physiological response to cold exposure is peripheral skin vasoconstriction which results in a reduction in skin blood flow. The afferent neural signaling pathway of cold stress traverses through the spinal cord to the preoptic area of the hypothalamus, with efferent signals traveling from the brain through the intermediolateral cell column of the spinal cord and to sympathetic nerves innervating cutaneous blood vessels. However, chronic cold exposure gradually leads to an attenuated vasoconstrictor response. ${ }^{5)}$ These habituation effects are unfavorable for the maintenance of body temperature, although the specific physiological mechanisms underlying the blunting of shivering and vasoconstrictor responses to cold exposure remain elusive.

Alteration of the set point for adrenergic and vagal outflow is observed in other cardiovascular disorders as well. Consequential excessive sympathoexcitation and vagal nerve withdrawal worsen the cardiac pathological conditions. For example, in heart failure due to impaired left ventricular function, attenuated heart rate variability and an impaired reflex vagal response to a rise in blood pressure occur, and the degree of baroreflex desensitization has been considered a prognostic factor for negative outcomes in patients with heart failure. ${ }^{67)}$ Chemical ablation of cardiac sympathetic afferents exhibits protective effects against deleterious cardiac remodeling in heart failure. ${ }^{8)}$ However, several experiments confirmed that the baroreceptor nerve input itself was not diminished in human heart failure and the baroreflex was responding appropriately to perceived decreases in stroke volume or blood pressure by eliciting sympathetic activation. ${ }^{9,10)}$ Increasing evidence of parasympathetic-sympathetic imbalance in heart failure rather focuses on the modulation of the central nervous system. ${ }^{11)}$

The dorsal medial nucleus of the tractus solitarius (dmNTS) in the brainstem comprises the primary central termination of baroreceptor and cardiac mechanosensor afferents (Figure). Subsequently, glutamatergic neurons in the NTS relay the baroreceptor input to GABAergic neurons in the caudal ventrolateral medulla (CVLM) and they inhibit neurons in the rostral ventrolateral medulla (RVLM), which regulate the tonus of sympathetic nerves as vasomotor center, to reduce sympathetic nerve activity. ${ }^{12)}$ Inputs from the NTS also transmit to the paraventricular nucleus (PVN) of the hypothalamus, and the PVN integrates autonomic responses with information from the forebrain such as the cerebral limbic system. Meanwhile, sympathetic afferents from cardiac chemoreceptors project to the vasomotor center of the brainstem and thalamus via the spinal cord.

It has been reported that the brain renin-angiotensin system (RAS) is activated in experimental chronic heart failure models. Expression of angiotensin II type 1 receptors (AT1R) increased in the NTS and RVLM of heart

From the ${ }^{1}$ Department of Cardiovascular Medicine, Graduate School of Medicine, The University of Tokyo, Tokyo, Japan and ${ }^{2}$ Department of Advanced Cardiology, The University of Tokyo, Tokyo, Japan.

Address for correspondence: Yukiteru Nakayama, MD or Katsuhito Fujiu, MD, Department of Cardiovascular Medicine, Graduate School of Medicine, The University of Tokyo, 7-3-1 Hongo, Bunkyo, Tokyo 113-8655, Japan. E-mail: ynakayama-tky@umin.ac.jp or fujiu-tky@umin.ac.jp

Received for publication November 30, 2018. Revised and accepted December 5, 2018.

doi: 10.1536/ihj.18-677

All rights reserved by the International Heart Journal Association. 


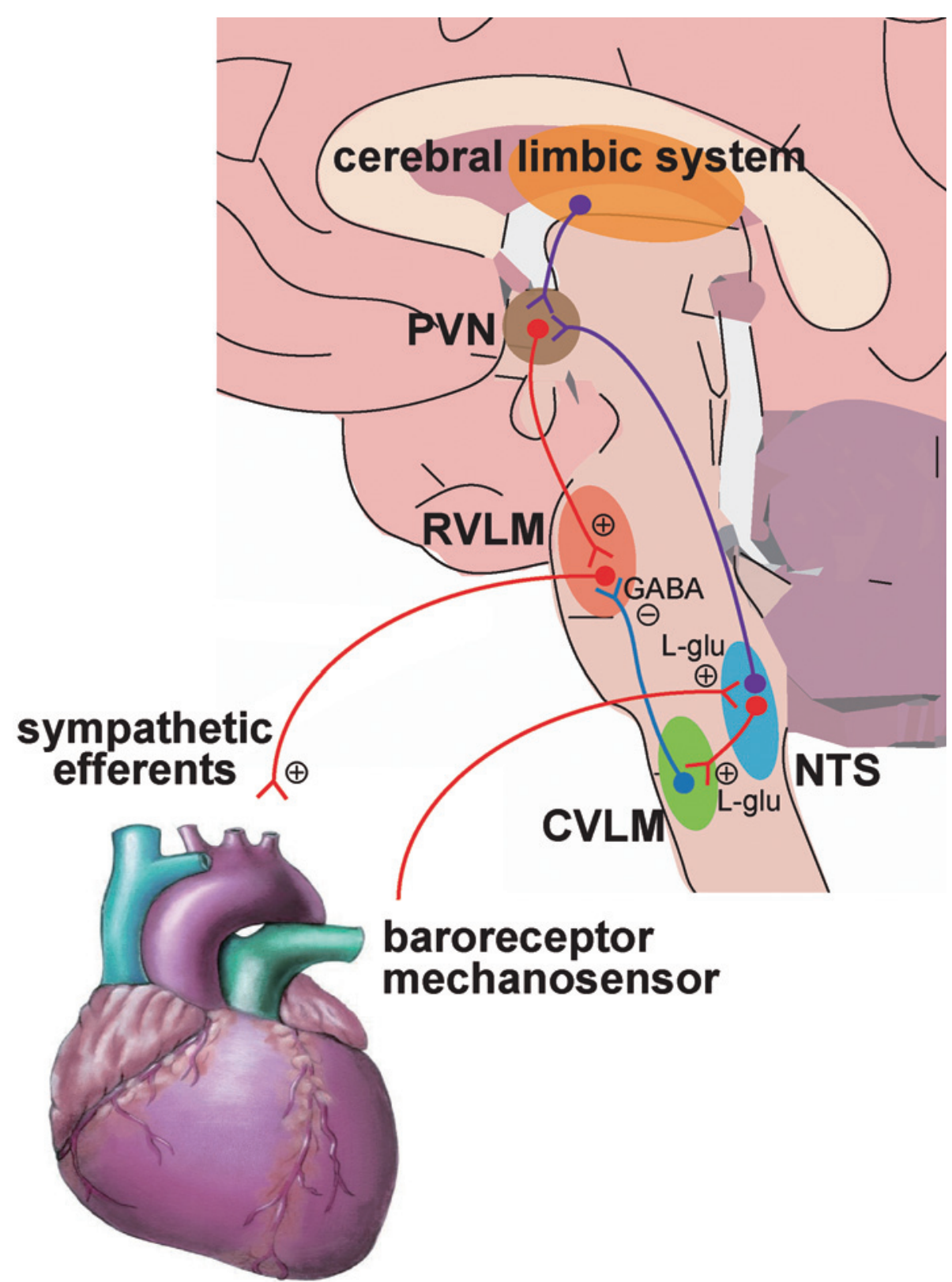

Figure. Neural circuits involved in autonomic nervous system in heart failure. Baroreceptor nerve inputs transmit to the vasomotor center in the brainstem, where the tonus of sympathetic nerves is set down after integration with information from the forebrain.

failure models via c-fos, p38, and ERK1/2 pathways, and augmented aldosterone was also shown to contribute to increased AT1R levels. ${ }^{13)}$ Upregulated AT1 signaling activates both the transcription factor activator protein-1 (AP1) and $\mathrm{NAD}(\mathrm{P}) \mathrm{H}$ oxidase. ${ }^{14)} \mathrm{AP}-1$ regulates inflammatory cytokines and $\mathrm{NAD}(\mathrm{P}) \mathrm{H}$ oxidase is implicated in the generation of reactive oxygen species (ROS). This modulation of the brainstem in heart failure is supported by the evidence that AT1R antagonists blocked the cascades. In the PVN as well, heart failure leads to AT1R expression at higher levels and upregulated RAS signaling, which induces inflammatory cytokines, activates NAD $(\mathrm{P}) \mathrm{H}$ oxidase to increase oxidative stress, ${ }^{15)}$ and impairs neuronal nitric oxide synthase (nNOS). NO production in the PVN has sympatho-inhibitory effects coupled with an increased expression of GABA and inhibitory action of glutamate, ${ }^{16)}$ while ROS enhances excitatory inputs through increasing glutamatergic and decreasing GABAergic transmission. Although accumulating evidence indicates a key role for inflammation in the sympathoexcitatory response, the direct underlying mechanism of the linkage has yet to be fully elucidated. Localized inflammatory cytokine expression activates microglia in the PVN, ${ }^{17}$ ) brain perivascular macrophages, ${ }^{18)}$ and astrocytes in RVLM. ${ }^{19)}$ These histo- 
logical remodelings could contribute to a shift of the set point. Structural alterations also occur in downward neural circuits. Inflammatory cytokines were induced in stellate ganglia and dorsal root ganglia after the acute phase of myocardial infarction, and highly elevated apoptotic activity and at the same time nerve sprouting and hyperinnervation were observed after chronic myocardial infarction. $^{20,21)}$

Numerous studies have explored the pivotal roles of brain RAS, NO, and inflammatory cytokines in the abnormal regulation of autonomic nerve activity in heart failure. Over a decade, new technologies including optogenetics and single cell transcriptomes have enabled further findings in the field of neuroscience. It has been only recently clarified that the mechanically activated ion channels PIEZO1 and PIEZO2 are together identified as aortic baroreceptors. ${ }^{22}$ The relationship between cardiovascular diseases and autonomic imbalance should be examined in greater detail in the future.

\section{Disclosures}

\section{Conflicts of interest: None.}

\section{References}

1. Mongkhonsiri P, Tong-un T, Wyss JM, Roysommuti S. Blunted nighttime sympathetic nervous system response to stress among Thai men with positive family history of sudden unexplained nocturnal death syndrome. Int Heart J 2019; 60: 55-62.

2. Tobaldini E, Brugada J, Benito B, et al. Cardiac autonomic control in Brugada syndrome patients during sleep: the effects of sleep-disordered breathing. Int J Cardiol 2013; 168: 3267-72.

3. Zheng J, Zheng D, Su T, Cheng J. Sudden unexplained nocturnal death syndrome: The hundred years' enigma. J Am Heart Assoc 2018; 7: e007837.

4. Chanavirut R, Tong-un T, Jirakulsomchok D, et al. Abnormal autonomic nervous system function in rural Thai men: A potential contributor to their high risk of sudden unexplained nocturnal death syndrome. Int J Cardiol 2017; 226: 87-92.

5. Castellani JW, Young AJ. Human physiological responses to cold exposure: Acute responses and acclimatization to prolonged exposure. Auton Neurosci 2016; 196: 63-74.

6. La Rovere MT, Bigger JT, Marcus FI, et al. Baroreflex sensitivity and heart-rate variability in prediction of total cardiac mortality after myocardial infarction. ATRAMI (Autonomic Tone and Reflexes After Myocardial Infarction) Investigators. Lancet 1998; 351: 478-84.
7. Mortara A, La Rovere MT, Pinna GD, et al. Arterial baroreflex modulation of heart rate in chronic heart failure. Circulation 1997; 96: 3450-8

8. Wang H-J, Wang W, Cornish KG, et al. Cardiac sympathetic afferent denervation attenuates cardiac remodeling and improves cardiovascular dysfunction in rats with heart failure. Hypertension 2014; 64: 745-55.

9. Floras JS. Sympathetic nervous system activation in human heart failure: clinical implications of an updated model. J Am Coll Cardiol 2009; 54: 375-85.

10. Floras JS, Ponikowski P. The sympathetic/parasympathetic imbalance in heart failure with reduced ejection fraction. Eur Heart J 2015; 36: 1974-82b.

11. Kishi T. Heart failure as a disruption of dynamic circulatory homeostasis mediated by the brain. Int Heart J 2016; 57: 145-9.

12. Mandel DA, Schreihofer AM. Glutamatergic inputs to the CVLM independent of the NTS promote tonic inhibition of sympathetic vasomotor tone in rats. Am J Physiol Heart Circ Physiol 2008; 295: H1772-9.

13. Huang BS, Zheng H, Tan J, et al. Regulation of hypothalamic renin-angiotensin system and oxidative stress by aldosterone. Exp Physiol 2011; 96: 1028-38.

14. Zucker IH, Schultz HD, Patel KP, et al. Regulation of central angiotensin type 1 receptors and sympathetic outflow in heart failure. Am J Physiol Heart Circ Physiol 2009; 297: H1557-66.

15. Kang Y-M, Ma Y, Elks C, et al. Cross-talk between cytokines and renin-angiotensin in hypothalamic paraventricular nucleus in heart failure: role of nuclear factor-kappaB. Cardiovasc Res 2008; 79: 671-8.

16. Zheng H, Liu X, Li Y, Patel KP. Gene transfer of neuronal nitric oxide synthase to the paraventricular nucleus reduces the enhanced glutamatergic tone in rats with chronic heart failure. Hypertension 2011; 58: 966-73.

17. Shi P, Diez-Freire C, Jun JY, et al. Brain microglial cytokines in neurogenic hypertension. Hypertension 2010; 56: 297-303

18. Yu Y, Zhang ZH, Wei SG, et al. Brain perivascular macrophages and the sympathetic response to inflammation in rats after myocardial infarction. Hypertension 2010; 55: 652-9.

19. Isegawa K, Hirooka Y, Katsuki M, et al. Angiotensin II type 1 receptor expression in astrocytes is upregulated leading to increased mortality in mice with myocardial infarction-induced heart failure. Am J Physiol Heart Circ Physiol 2014; 307: H1448-55

20. Gao C, Howard-Quijano K, Rau C, et al. Inflammatory and apoptotic remodeling in autonomic nervous system following myocardial infarction. PLoS One 2017; 12: e0177750.

21. Nguyen BL, Li H, Fishbein MC, et al. Acute myocardial infarction induces bilateral stellate ganglia neural remodeling in rabbits. Cardiovasc Pathol 2012; 21: 143-8.

22. Zeng WZ, Marshall KL, Min S, et al. PIEZOs mediate neuronal sensing of blood pressure and the baroreceptor reflex. Science 2018; 362: 464-7. 